\title{
Transferência tendínea do grande dorsal com enxerto tendíneo homólogo para as lesões irreparáveis do manguito rotador: técnica cirúrgica*
}

\section{Latissimus Dorsi Tendon Transfer using Tendinous Allograft for Irreparable Rotator Cuff Lesions: Surgical Technique}

\author{
Alberto Naoki Miyazaki ${ }^{1}$ Caio Santos Checchia1® Wagner de Castro Lopes ${ }^{1}$ \\ João Manoel Fonseca Filho ${ }^{1}$ Guilherme do Val Sella ${ }^{1}$ Luciana Andrade da Silva ${ }^{1}$ \\ ${ }^{1}$ Grupo de Cirurgia de Ombro e Cotovelo, Departamento de \\ Ortopedia e Traumatologia, Faculdade de Ciências Médicas da Santa \\ Casa de São Paulo, São Paulo, Brasil \\ Rev Bras Ortop 2019;54:99-103. \\ Address for correspondence Caio Santos Checchia, Grupo de Cirurgia \\ de Ombro e Cotovelo, Departamento de Ortopedia e Traumatologia, \\ Faculdade de Ciências Médicas da Santa Casa de São Paulo, São Paulo, \\ SP, Brasil (e-mail: caio.checchia@gmail.com).
}

\section{Resumo \\ Palavra-chave \\ - lesões do manguito rotador \\ - transferência tendinosa \\ - lesões do ombro \\ - ombro \\ - ortopedia}

No contexto do tratamento cirúrgico dos pacientes jovens com lesões irreparáveis da porção posterossuperior do manguito rotador, a técnica mais usada é a transferência do tendão do grande dorsal para a porção superolateral do tubérculo maior, conforme descrita e preconizada por Gerber et al. Entretanto, duas características dessa técnica podem levar a resultados ruins e complicações: (i) a deiscência da origem do deltoide, que ocorre devido à sua violação durante a criação da via em golpe de sabre e (ii) a ruptura pós-operatória da inserção da transferência. Na tentativa de solucionar esses dois problemas, as seguintes modificações foram feitas à técnica cirúrgica original. Por meio de uma única via deltopeitoral, o tendão do grande dorsal é isolado e desinserido do úmero. Ele é então alongado e reforçado com um enxerto tendíneo homólogo, transferido ao redor do úmero e fixado à porção superolateral do tubérculo maior. Não foi usada imobilização pós-operatória com órtese toracobraquial rígida.

Latissimus dorsi transfer around the shoulder is the most frequently used surgical technique to treat young patients with irreparable posterosuperior rotator cuff lesions. This technique, as initially described and popularized by Gerber et al., has two main drawbacks that may predispose to complications and unsatisfactory functional results: 1) postoperative rupture of the origin of the deltoid, as its detachment from the acromion is necessary during the superior approach to the shoulder; and 2) postoperative rupture of the transferred tendon. In an attempt to avoid these problems, the authors have developed the following modifications to the original technique. Through a deltopectoral approach, the latissimus dorsi tendon is identified and detached from the humerus shaft. After being reinforced and elongated with a tendinous allograft, it is transferred around the humerus and fixed to the superolateral aspect of the greater tubercle. No rigid thoraco-brachial immobilization is used postoperatively.

\footnotetext{
Trabalho desenvolvido no Departamento de Ortopedia e Traumatologia, Faculdade de Ciências Médicas da Santa Casa de São Paulo (DOT-FCMSCSP), São Paulo, SP, Brasil.

(D)Caio Santos Checchia's ORCID is https://orcid.org/0000-00031562-9795.
}

received

July 7, 2017

accepted

September 28, 2017
DOI https://doi.org/

10.1055/s-0038-1676989. ISSN 0102-3616.
Copyright $(\odot 2019$ by Sociedade Brasileira License terms de Ortopedia e Traumatologia. Published by Thieme Revnter Publicações Ltda, Rio de Janeiro, Brazil 


\section{Introdução}

As lesões tendíneas crônicas do manguito rotador podem levar a atrofia, degeneração gordurosa e alterações funcionais de seus respectivos músculos. ${ }^{1}$ Quando acometem a região posterossuperior do manguito, na maioria das vezes resultam em dor, diminuição da força de rotação lateral e de elevação do ombro, além da dificuldade de posicionamento da mão no espaço. ${ }^{1-3}$

Diversas técnicas cirúrgicas foram descritas com o intuito de melhorar a qualidade de vida dos pacientes com lesões irreparáveis desses tendões. Especialmente na população mais jovem (com menos de 60 anos) e com maior demanda funcional, a transferência do tendão do músculo grande dorsal (TGD) para o tubérculo maior (inicialmente descrita por Gerber, ${ }^{1}$ em 1992) é a opção terapêutica mais comumente usada. ${ }^{4}$ Diversos trabalhos demonstram que com ela é possível a obtenção de bons resultados no combate à dor e à disfunção. ${ }^{3,4}$ Entretanto, até 36\% de insucessos foram encontrados. Somado a isso, ainda são escassas e controversas as investigações sobre os fatores preditivos de maus resultados. ${ }^{2,3}$

Há evidências, porém, de que a maioria dos insucessos ocorra por um dos dois seguintes motivos: (i) deiscência da reinserção da transferência ou (ii) deiscência da origem do músculo deltoide. ${ }^{5}$ Dessa maneira, alguns autores recentemente propuseram mudanças na técnica original de Gerber. O uso da artroscopia ${ }^{6}$ ou de somente uma via de acesso, ${ }^{7}$ por exemplo, foi preconizado para evitar a violação da inserção proximal do deltoide. Já a desinserção do grande dorsal com a manutenção de alguns fragmentos ósseos da cortical umeral junto ao tendão foi preconizada para reforçar a cicatrização da transferência junto ao tubérculo maior. ${ }^{5}$
Não encontramos na literatura, porém, técnica alguma que se preocupasse em evitar simultaneamente essas duas complicações. É justamente com esse intuito, então, que propomos as seguintes modificações à técnica cirúrgica: o tendão do grande dorsal é alongado e reforçado com um enxerto tendíneo, permitindo assim o uso de uma única via de acesso deltopeitoral.

O objetivo do presente trabalho é descrever as modificações propostas à técnica cirúrgica.

\section{Descrição da Técnica}

O paciente é colocado em posição de cadeira de praia. Através de uma via deltopeitoral, identifica-se a lesão irreparável do manguito rotador. Para a identificação do nervo axilar, do nervo radial e da inserção dos tendões dos músculos grande dorsal e redondo maior ( - Fig. 1), o tendão conjunto deve ser afastado medialmente e o tendão do peitoral maior, distalmente (às vezes, faz-se necessária a tenotomia do terço proximal da sua inserção, que então deve ser reinserida no fim da cirurgia). O tendão do grande dorsal é reparado, totalmente desinserido do úmero e seu ventre muscular é parcialmente dissecado. Ele é então alongado e reforçado com um enxerto tendíneo homólogo que é recortado para ficar com a mesma largura do tendão do paciente e com comprimento suficiente para alcançar o tubérculo maior (-Fig. 2A e B).

Com uma pinça longa e curva passada subperiostealmente entre o úmero e as cabeças longa e lateral do tríceps (-Fig. 2A), cria-se um túnel através do qual se passa o tendão (já alongado) para a porção posterior do úmero. Por fim, com o ombro colocado em 15 graus de abdução e 60 graus de rotação lateral e com o tendão tracionado de forma a não ficar

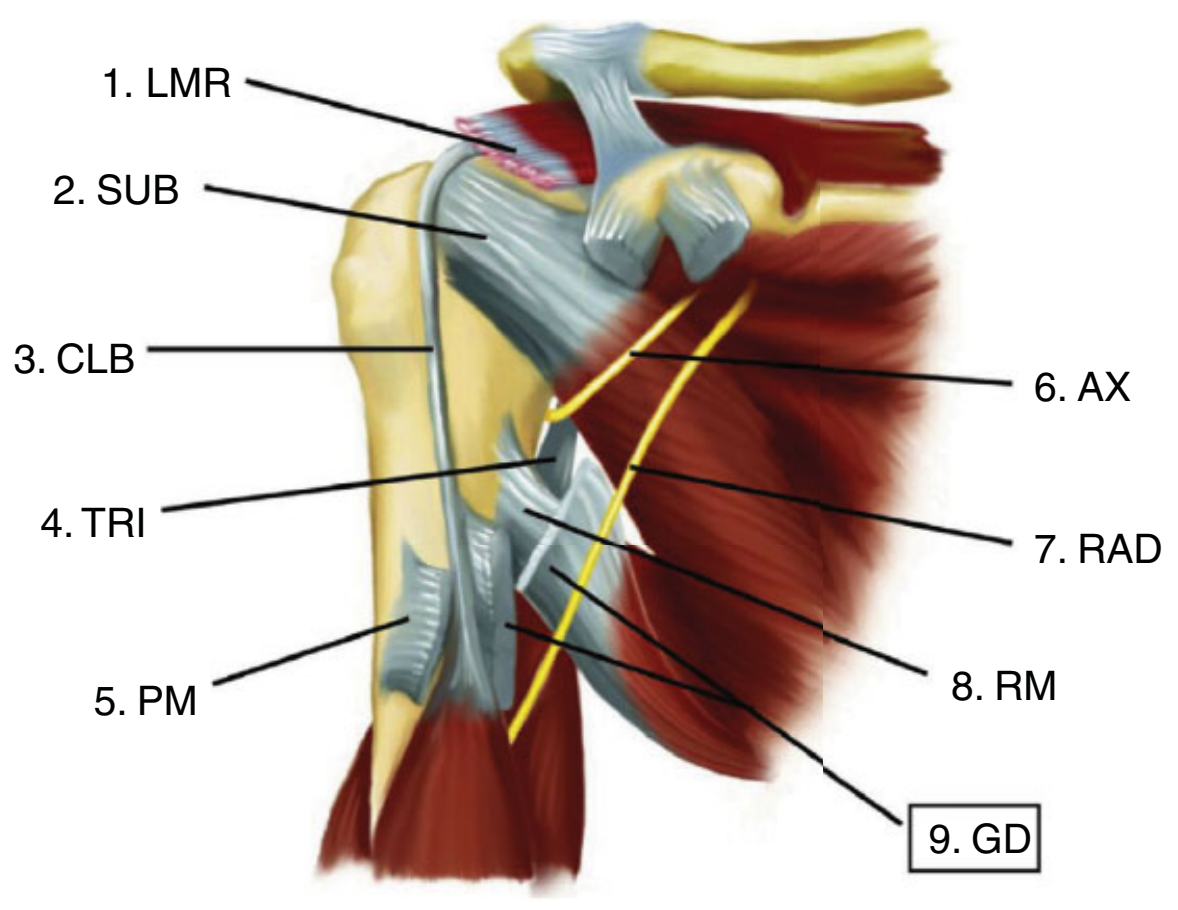

Fig. 1 Ilustração de um ombro direito, visto de frente, sem o deltoide e os músculos do tendão conjunto. Abreviações: AX, nervo axilar; CLB, cabeça longa do bíceps braquial; GD, tendão (já isolado e desinserido) do músculo grande dorsal; LMR, lesão do manguito rotador; PM, inserção tendínea do peitoral maior; RAD, nervo radial; RM, inserção tendínea do musculo redondo maior; SUB, tendão do subescapular; TRI, cabeça longa do músculo tríceps braquial. 


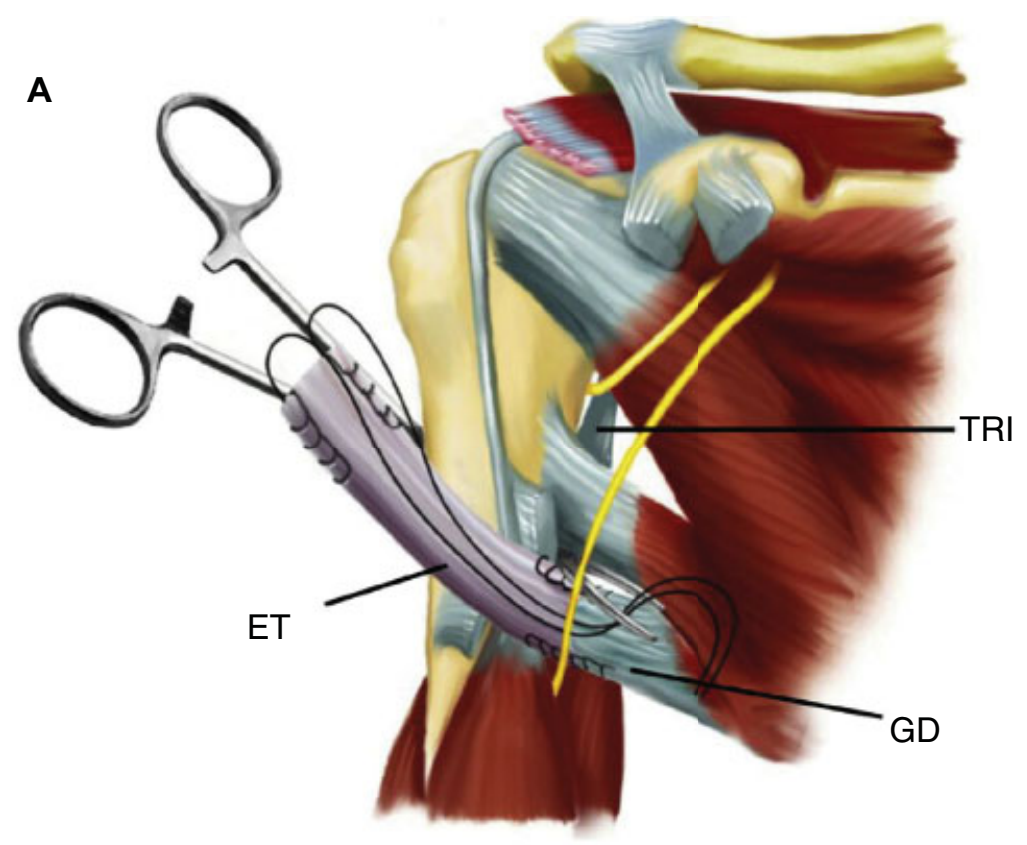

B

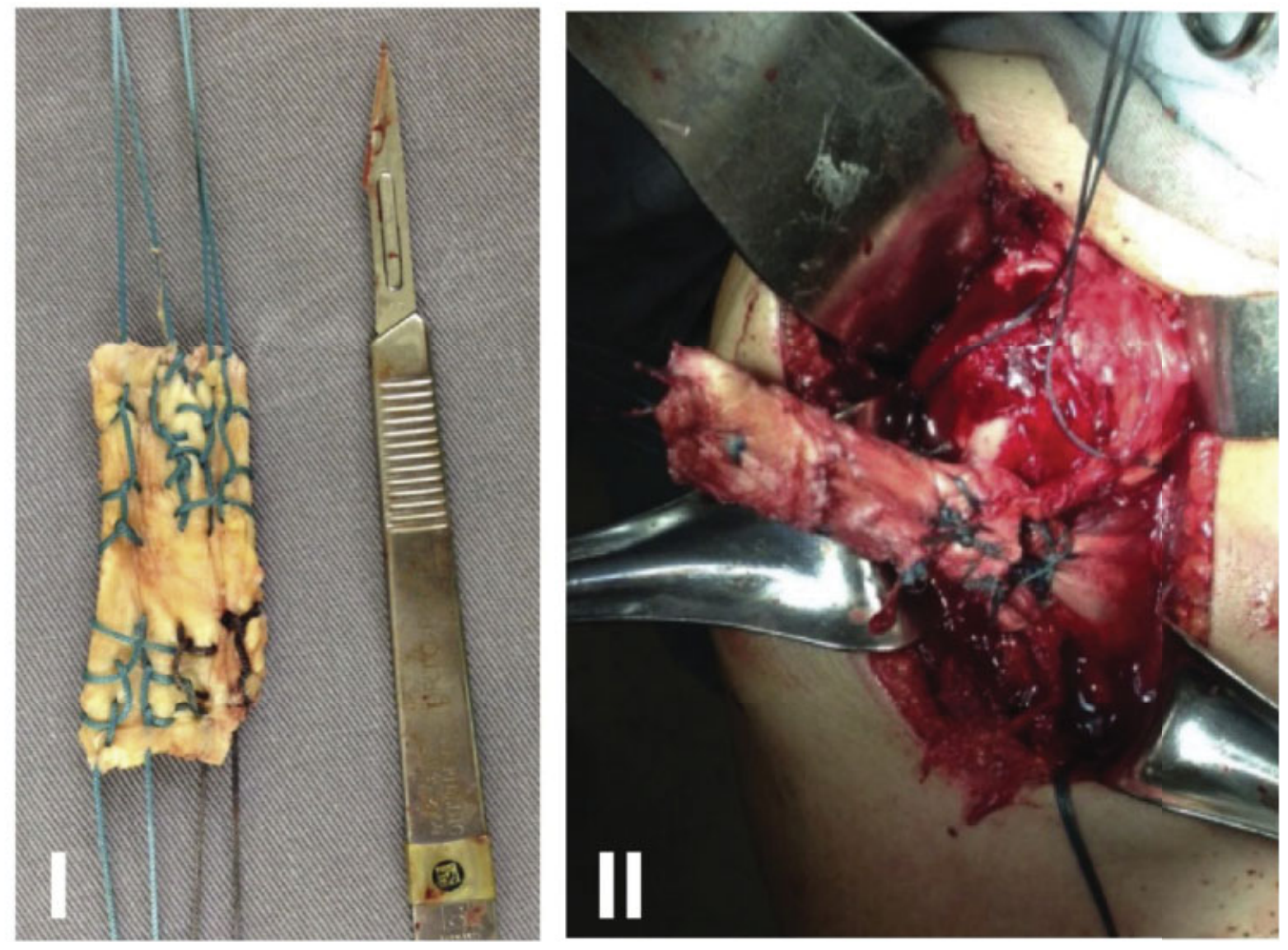

Fig. 2 (A) neste passo da cirurgia, o enxerto tendíneo (ET) já foi suturado ao tendão do grande dorsal (GD) e a pinça já foi passada ao redor do úmero (entre a porção proximal da sua diáfise e os ventres musculares das cabeças lateral e longa do tríceps braquial (TRI). (B) Enxerto homólogo de tendão calcâneo já preparado, antes (I) e após (II) sua sutura ao tendão do grande dorsal (antes de serem passados ao redor do úmero).

frouxo, a transferência é suturada (com fio resistente e não absorvível) à porção súperolateral do tubérculo maior (-Fig. 3A e B). Preferencialmente, como preconiza Gerber, as suturas mais anteriores devem englobar também o tendão do subescapular (elas podem até ser usadas para reparo de lesões proximais do subescapular, caso estejam presentes).Já as suturas mais mediais podem ser usadas para suturar 0 restante do coto do manguito à transferência ( - Fig. $3 \mathbf{3 A}$ ). 
A

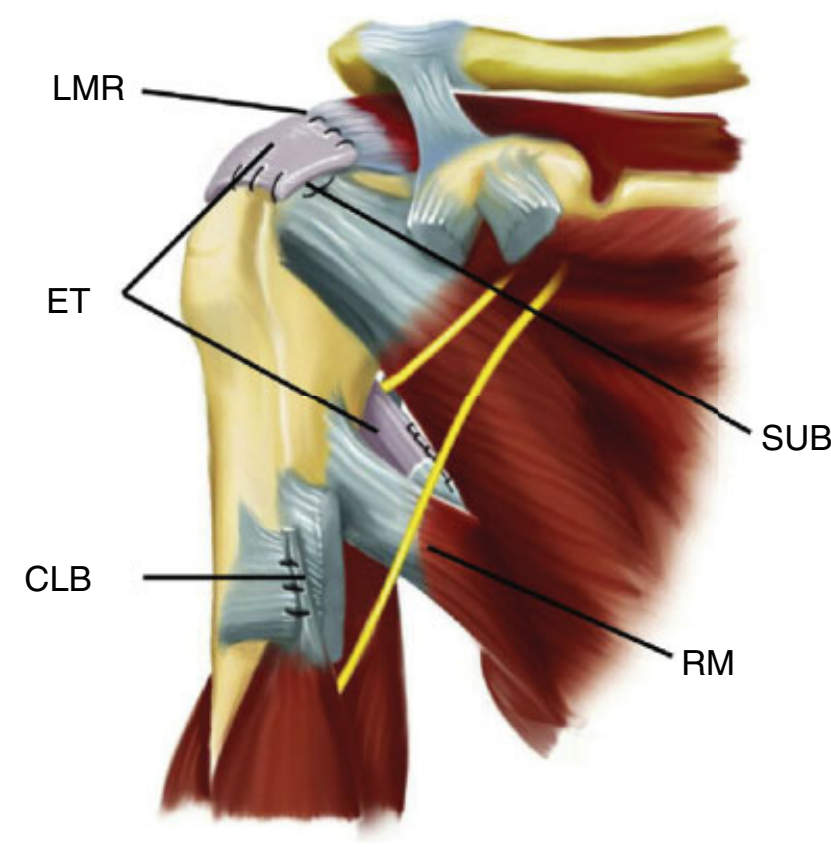

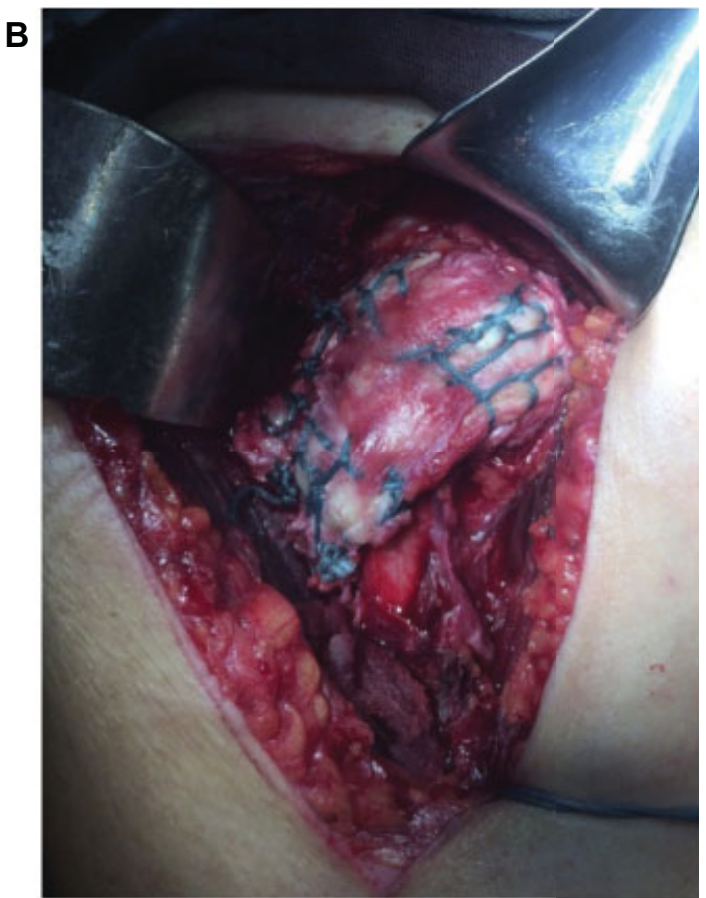

Fig. 3 Na última etapa da cirurgia, o tendão transferido do grande dorsal (GD), já alongado e reforçado pelo enxerto tendíneo (ET), é suturado à porção superolateral do tubérculo maior, se possível ao coto remanescente do manguito rotador (LMR) e à porção proximal do tendão do subescapular (SUB). Note que, após a transferência, o tendão do grande dorsal fica posterior ao do redondo maior (RM). Note também que, nesta ilustração, a cabeça longa do bíceps (CLB) foi tenotomizada e tenodesada ao tendão do peitoral maior.

Ao término da cirurgia, o paciente é imobilizado em uma tipoia de abdução em rotação neutra por seis semanas. Após esse período, são iniciados exercícios de ganho e manutenção da ADM de todos os movimentos do ombro, porém ainda sem fortalecimento. Esses são iniciados após quatro meses da cirurgia.

\section{Comentários Finais}

Na eventual falha da transferência tendínea no tratamento dessas lesões, a artroplastia reversa provavelmente é a única forma terapêutica suficientemente embasada na literatura capaz de proporcionar melhoria da dor e da função do ombro. Para isso, porém, ela requer um deltoide funcionante, já que nessa situação ele é a única unidade motora capaz de gerar vetores que resultem na elevação articular. ${ }^{2}$ Dessa maneira, pode-se argumentar que seja extremamente prudente evitar sua violação cirúrgica, conforme a feita na via de sabre aplicada na técnica descrita por Gerber ${ }^{1}$, por exemplo.

Só existem três opções para a via de acesso, portanto: uma única via de acesso posterior, uma via axilar e uma única via de acesso anterior. A via posterior permite boa dissecção do ventre muscular do grande dorsal, porém sua desinserção isolada (sem a desinserção do redondo maior) é difícil (já que seu tendão é anterior ao do redondo maior). Além disso, essa via não permite a identificação e o tratamento de eventuais lesões dos tendões do subescapular e da cabeça longa do bíceps.

A via de acesso axilar não permite a tentativa de um eventual reparo primário do manguito rotador, que sempre deve ser tentado antes de se fazer a transferência tendínea. Além disso, representa risco de graves lesões neurovascula- res devido à proximidade com as estruturas do plexo braquial e vasos axilares.

Por fim, há a via de acesso escolhida: uma única via de acesso deltopeitoral. Além de ser uma via de costume para os cirurgiões de ombro, ela permite a tentativa de reparo primário do manguito rotador (-Fig. 1), possibilita a desinserção isolada com facilidade do grande dorsal (-Fig. 1), permite facilmente a identificação e o isolamento dos nervos axilar e radial (-Fig. 1) e possibilita o tratamento de eventuais lesões dos tendões da cabeça longa do bíceps e do subescapular (-Fig. 1). Sua única desvantagem é a impossibilidade de se dissecar o ventre muscular do grande dorsal e, portanto, impede que seja tracionado até a porção superior do grande dorsal. Entretanto, dado que o tendão desse músculo é extremamente fino e friável, preconiza-se que sua inserção ao tubérculo maior deva ser de alguma forma reforçada (sua desinserção pós-cirúrgica é até um dos poucos fatores preditivos de mau resultado funcional estatisticamente provados, ${ }^{2}$ além de haver evidência de que seu reforço com a incorporação de pequenos fragmentos ósseos diminui esse risco ${ }^{6}$ ). Portanto, o uso do enxerto tendíneo pode conseguir de uma só vez solucionar esses dois problemas: ele alonga o tendão que será transferido (permite o uso de uma única via anterior) e reforça a inserção do tendão ao tubérculo maior (possivelmente diminui o risco de desinserção pós-cirúrgica). Ficamos tão seguros da firmeza do reforço durante a cirurgia que até permitimos que a imobilização pós-operatória seja feita com uma tipoia de abdução, e não com uma órtese toracobraquial rígida.

Vale ressaltar que a associação de uma via anterior a uma posterior permitiria a dissecção e o alongamento adequados 
A

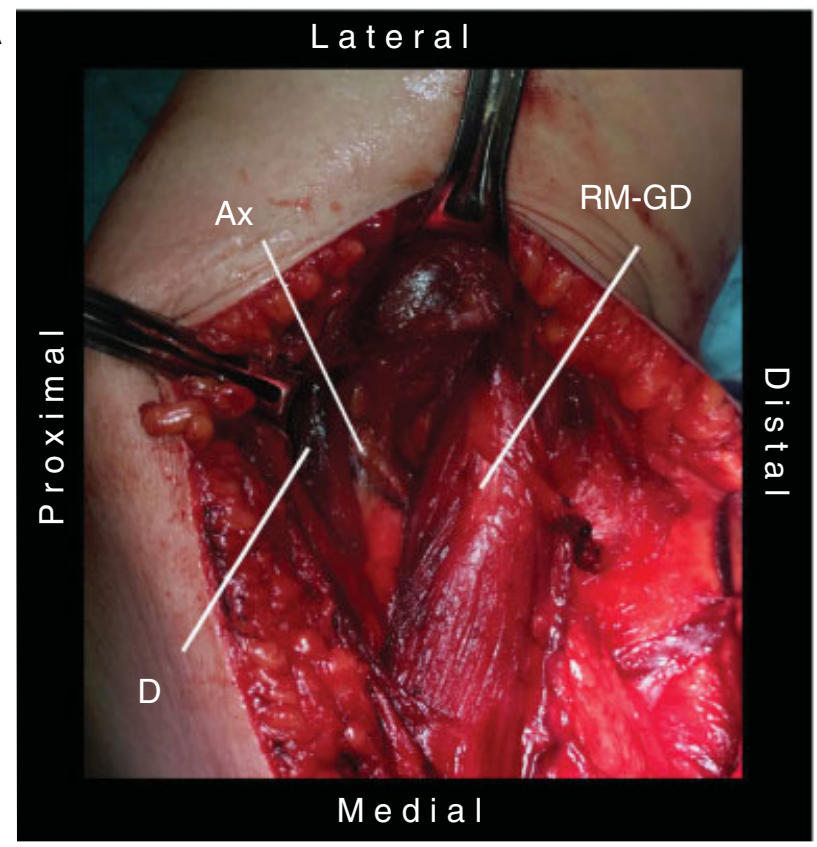

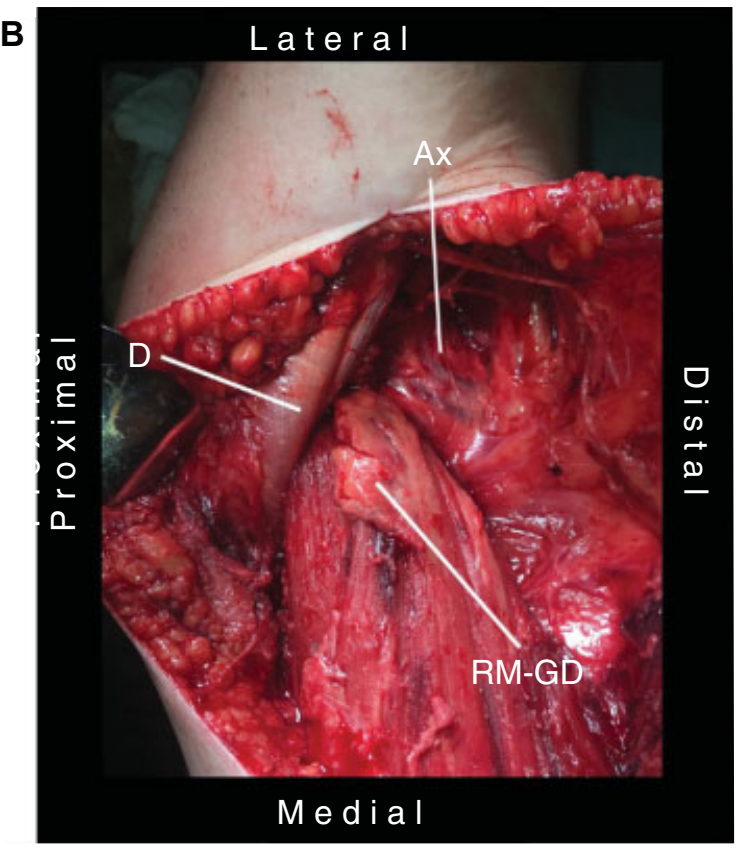

Fig. 4 Ombro direito. Vista posterior. Técnica convencional, conforme a proposta por Gerber. ${ }^{1}$ Note a relação antes (A) e após (B) a transferência do redondo maior (RM) e do grande dorsal (GD) em relação ao nervo axilar e vasos circunflexos umerais posteriores (Ax). D, deltoide.

do grande dorsal, mas não seria capaz, por si só, de prover um reforço à inserção tendínea. Essa associação, portanto, parece ser menos vantajosa do que a técnica aqui proposta.

Por fim, há quatro preocupações inerentes às modificações. A primeira envolve a criação de um novo ponto de fragilidade na transferência, que é o local de sutura entre o enxerto e o tendão nativo. A segunda é referente à capacidade de cicatrização do enxerto ao osso. Isso pois há alguma evidência ${ }^{8}$ de que em longo prazo, quando comparado ao autólogo, o enxerto homólogo demore mais para remodelar e possivelmente proporcione menor resistência mecânica.

A terceira preocupação é referente à possibilidade de que o tendão do grande dorsal seja alongado demais, diminua em demasia a força final resultante da unidade musculotendínea transferida sobre o ombro. Essas três possibilidades só poderão ser confirmadas ou descartadas após seguimento pós-operatório em longo prazo.

Já a quarta preocupação é que a transferência, quando feita conforme a técnica aqui descrita, tem relações topográficas (com as demais estruturas anatômicas do ombro) diferentes das relações da técnica proposta por Gerber. ${ }^{1}$ Uma delas é que o tendão transferido, ao invés de posterior, fica anterior ao tríceps (entre ele e o úmero) (-Fig. 3A) e isso eventualmente pode propiciar a formação de aderências fibrosas. A outra é que o tendão passa inferior, ao invés de superior, ao nervo axilar e aos vasos circunflexos umerais posteriores (-Fig. 4). Não fizemos seguimento em longo prazo, mas podemos informar que uma análise parcial e precoce de dez pacientes operados com essa técnica não demonstrou qualquer alteração neurovascular durante exame físico.
Conflitos de Interesse

Os autores declaram não haver conflitos de interesse.

\section{Referências}

1 Gerber C. Latissimus dorsi transfer for the treatment of irreparable tears of the rotator cuff. Clin Orthop Relat Res 1992;(275): $152-60$

2 Gerber C, Rahm SA, Catanzaro S, Farshad M, Moor BK. Latissimus dorsi tendon transfer for treatment of irreparable posterosuperior rotator cuff tears: long-term results at a minimum follow-up of ten years. J Bone Joint Surg Am 2013;95(21):1920-6

3 El-Azab HM, Rott O, Irlenbusch U. Long-term follow-up after latissimus dorsi transfer for irreparable posterosuperior rotator cuff tears. J Bone Joint Surg Am 2015;97(6):462-9

4 Aoki M, Okamura K, Fukushima S, Takahashi T, OginoT. Transfer of latissimus dorsi for irreparable rotator-cuff tears. J Bone Joint Surg Br 1996;78(5):761-6

5 Tauber M, Moursy M, Forstner R, Koller H, Resch H. Latissimus dorsi tendon transfer for irreparable rotator cuff tears: a modified technique to improve tendon transfer integrity: surgical technique. J Bone Joint Surg Am 2010;92(Suppl 1 Pt 2):226-39

6 Gervasi E, Causero A, Parodi PC, Raimondo D, Tancredi G. Arthroscopic latissimus dorsi transfer. Arthroscopy 2007;23(11):1243. e1-e4

7 Habermeyer P, Magosch P, Rudolph T, Lichtenberg S, Liem D. Transfer of the tendon of latissimus dorsi for the treatment of massive tears of the rotator cuff: a new single-incision technique. J Bone Joint Surg Br 2006;88(2):208-12

8 Scheffler SU, Schmidt T, Gangéy I, Dustmann M, Unterhauser F, Weiler A. Fresh-frozen free-tendon allografts versus autografts in anterior cruciate ligament reconstruction: delayed remodeling and inferior mechanical function during long-term healing in sheep. Arthroscopy 2008;24(4):448-58 